\title{
Tear ferning in normal dogs and dogs with keratoconjunctivitis sicca
}

\author{
David Williams* and Heather Hewitt \\ Department of Veterinary Medicine, University of Cambridge, Madingley Road, Cambridge CB3 OES, UK
}

\begin{abstract}
This study evaluates tear ferning as an ancillary technique for the evaluation of the canine tear film in normal eyes and eyes affected by keratoconjunctivitis sicca (KCS). Thirty dogs with KCS and 50 control dogs with normal tear film were evaluated with a full ophthalmoscopic examination and a Schirmer tear test type 1 (STT) determined before tear samples were obtained from the medial canthus with a microhaematocrit capillary tube. 10ul of tear was placed on a microscope slide and the time to first formation of a fern of crystallised tear solute was determined. The appearance of the ferning pattern was graded and correlated with the STT value. All eyes with KCS had abnormal ferning patterns while 39 out of the 50 normal dogs (78\%) had so-called 'normal' ferning patterns. The mean STT for dogs showing 'normal' ferning patterns was $20.6 \mathrm{~mm} / \mathrm{min}$ for the left eye and $21.3 \mathrm{~mm} / \mathrm{min}$ for the right eye. STT values for eyes with 'abnormal' ferning patterns were $10.9 \mathrm{~mm} / \mathrm{min}$ and $12.4 \mathrm{~mm} / \mathrm{min}$, these differing from the normal eyes with STT above $15 \mathrm{~mm} / \mathrm{min}$ significantly. These findings suggest that tear ferning could be a valuable technique for assessment of the tear film in dogs with KCS.
\end{abstract}

Keywords: Dog, Dry eye, Ferning, Keratoconjunctivitis sicca, Tear.

\section{Introduction}

The phenomenon of crystal formation in a fern-like pattern in drying solutions of body fluids has been recognised for several years. First described in human cervical mucus in the context of cervical smear (Papanicolau, 1946), ferning has been reported in other fluids from saliva (Guida et al., 1993), serum (Reece et al., 1984), nasal mucus (Ullery et al., 1959) as well as tears (Rolando, 1984).

In 1986 Rolando and colleagues devised a grading system for tear ferning (Rolando et al., 1986), dividing the ferning into one of four grades depending on the extent and pattern of the ferns. Rolando's four grades are detailed in Table 1.

One paper has demonstrated that tear ferning occurs in tears from dogs but only in the context of evaluating tear formation in canine eyes rendered tear deficient experimentally and treated with nerve growth factor (Coassin et al., 2005).

Silva and colleagues have documented tear ferning in 30 horses (Silva et al., 2016) and sought to quantify the ferning pattern with a computerised stereology tool. No detailed study of tear ferning in normal dogs and dogs with naturally occurring keratoconjunctivitis sicca has to date been published and thus it is our aim here to provide such a report.

Keratoconjunctivitis sicca (KCS) is a common ophthalmic disease in the dog (Williams, 2008) in which the Schirmer tear test (STT) is the standard diagnostic test (Gelatt et al., 1975).
Table 1. Classification of human tear ferning patterns (Rolando et al., 1986).

\begin{tabular}{cl}
\hline $\begin{array}{c}\text { Tear fern } \\
\text { grade }\end{array}$ & \multicolumn{1}{c}{ Description of ferning pattern } \\
\hline I & $\begin{array}{l}\text { Uniform arborisation in the entire field of } \\
\text { observation without spaces among the ferns, } \\
\text { with single ferns big and close branching }\end{array}$ \\
& $\begin{array}{l}\text { Ferning phenomenon still abundant but the } \\
\text { single ferns are smaller and with lower } \\
\text { frequency of branching compared with type }\end{array}$ \\
II & $\begin{array}{l}\text { I. Empty spaces begin to appear among the } \\
\text { ferns }\end{array}$ \\
& $\begin{array}{l}\text { Arborization of mucus is partially present. } \\
\text { Single ferns are little and incompletely } \\
\text { formed with no or rare branching. Large } \\
\text { spaces without ferning are present in the } \\
\text { field, including conglomerates of mucus } \\
\text { without any sign of organization }\end{array}$ \\
& $\begin{array}{l}\text { Ferning phenomenon is absent. The } \\
\text { specimen collected does not show any } \\
\text { organization and the mucus appears in } \\
\text { clusters and threads, which represent } \\
\text { possibly contaminated and degenerated } \\
\text { mucus mixed with exfoliated cells }\end{array}$ \\
\hline IV &
\end{tabular}

A number of other criteria are important in determining the ocular pathology in KCS from Rose Bengal staining, tear osmolality and tear ferning, these being considered central to a diagnosis of KCS in human patients to a greater degree than in veterinary ophthalmology (Van Bijsterveld, 1990). 
The use of tests such as tear ferning is not essential to a diagnosis of canine KCS but can assist in the evaluation of dogs with the condition, especially in animals with qualitative rather than quantitative tear film deficiency, in which STT may be normal but ocular surface pathology occurs through mucin or tear lipid deficiency with consequent increased tear evaporation.

We have shown in this study that animals with KCS and STT values below $10 \mathrm{~mm} / \mathrm{min}$ tended to have abnormal ferning patterns (types III or IV) while animals with STT above $10 \mathrm{~mm} / \mathrm{min}$ tended to have normal tear ferning patterns (types I or II) and demonstrated that tear ferning can be a valuable additional assessment of the properties of the tear film to supplement but not replace the Schimer tear test.

\section{Materials and Methods}

Eighty dogs examined at the Queen's Veterinary School Hospital, University of Cambridge were involved in the study described herein. Animals included 30 with $\mathrm{KCS}$ and 50 included as normal control animals. Dogs with KCS were evaluated prior to treatment with tear replacement medication or topical cyclosporine. The control animals were matched to the clinical cases as closely as possible for age, gender and breed but this was not always possible. All animals underwent a full ophthalmic evaluation using direct and indirect ophthalmoscopy and slit lamp biomiocroscopy before undergoing a Schirmer tear test 1. After these examinations tear fluid was obtained as previously described (Norn, 1988). Over $10 \mu \mathrm{l}$ of tear fluid was obtained from the medial canthus with a glass capillary tube (Fig. 1).

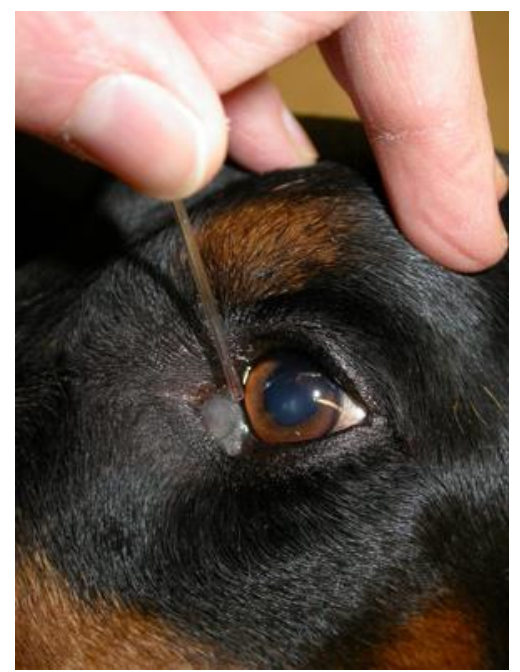

Fig. 1. Sampling of tear fluid from the medial canthus in a dog with mild KCS.

The order of sampling, i.e. using left or right eye first was randomised using an online random number table (http://stattrek.com/statistics/random-numbergenerator.aspx). An aliquot of $10 \mu \mathrm{l}$ of the fluid thus acquired was drawn up using a Gilson pipette and applied to a clean microscope slide kept at $20 \pm 2 \mathrm{oC}$ and $30 \pm 2 \%$ humidity. The slide was viewed by phase contrast microscopy at $\mathrm{x} 40$ magnification until all tear fluid had evaporated. The time taken to the earliest fern formation was determined and the final form of the tear fern was photographed after 5 minutes, by which time the slide was dry in all cases. The temperature and humidity at the point of sample taking and drop evaluation was determined using a LCD Digital Temperature Humidity 88 Meter Thermometer (DTYHTC8, White Industries, Reading UK).

A Fishers exact test was performed on the data to compare the ferning patterns of dogs with normal tear production and KCS (STT $<10 \mathrm{~mm} / \mathrm{min})$. A student $\mathrm{t}$ test was used to compare time to fern formation for dogs with different ferning patterns after Levene's test for equality of variance was performed. Data from right and left eyes was compared using a paired samples $\mathrm{T}$ test. Data analysis of fern type and time to fern formation was performed separately for the left and right eyes to avoid the conundrum of over-estimating the population size by using the data from both eyes together (Newcombe and Duff, 1987).

\section{Results}

The signalment of the dogs in this study in summarised in Table 2.

Table 2. Signalment of animals in KCS and control groups.

\begin{tabular}{lcc}
\hline & KCS group & Control group \\
\hline $\begin{array}{l}\text { Number of } \\
\text { animals }\end{array}$ & 30 & 50 \\
$\begin{array}{l}\text { Mean age of } \\
\text { animals }\end{array}$ & $12.2 \pm 3.4$ years & $11.4 \pm 2.2$ years \\
$\begin{array}{l}\text { Percentage of } \\
\text { KCS-predisposed } \\
\text { breeds }\end{array}$ & 50 & 56 \\
$\begin{array}{l}\text { Mean STT value } \\
\text { for both eyes }\end{array}$ & $6.2 \pm 3.5 \mathrm{~mm} / \mathrm{min}$ & $16.2 \pm 5.6 \mathrm{~mm} / \mathrm{min}$ \\
\hline
\end{tabular}

KCS patients were predominantly breeds predisposed to the condition (English Cocker spaniel 7 cases, West Highland white terrier 4 cases, Cavalier King Charles spaniel 2 cases, Yorkshire terrier 2 cases, Lhasa Apso 1 case) and a similar proportion of control animals were chosen from the same breeds to avoid inter-breed differences providing uncontrolled variation. The four types of tear ferning, as delineated by Rolando et al. (1986) are shown in Figure 2.

All 30 of the dogs with KCS had ferning patterns of types 3 and 4 while 39 out of the 50 normal dogs (78\%) had ferning patterns of types 1 and 2 . The mean STT for dogs showing normal (types 1 and 2) ferning patterns was $20.6 \mathrm{~mm} / \mathrm{min}$ for the left eye and $21.3 \mathrm{~mm} / \mathrm{min}$ for the right eye, these two populations of STT values not being statistically different. 


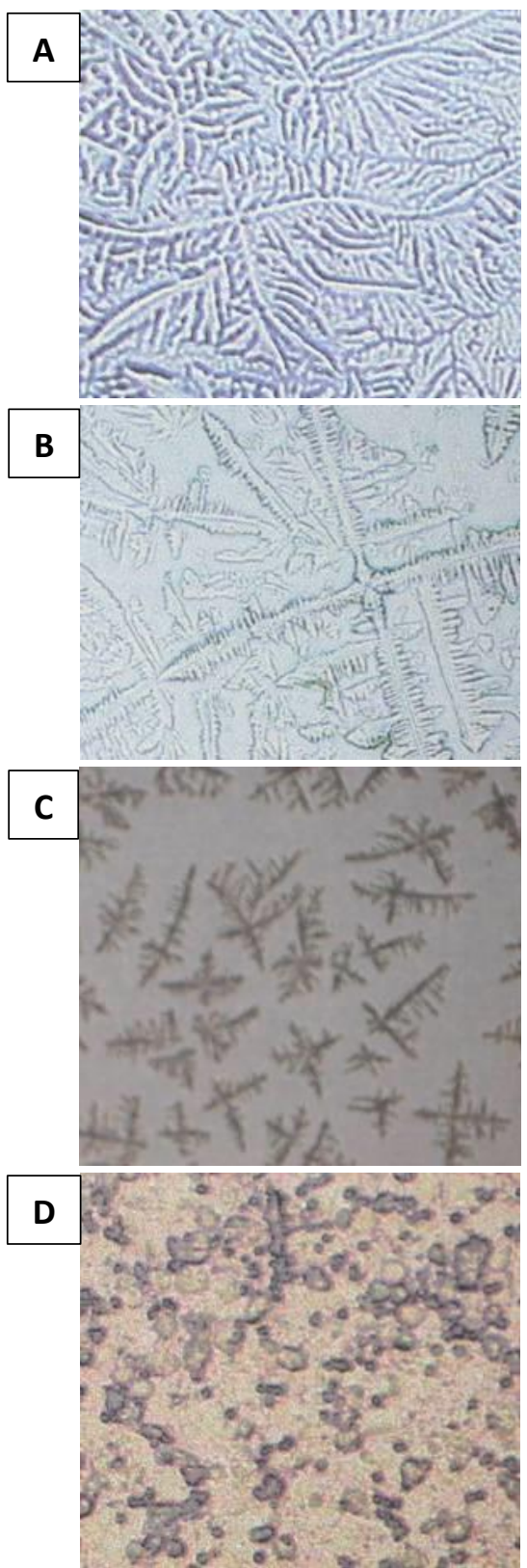

Fig. 2. The four grades of tear ferning taken at 20x magnification. (A): Tear ferning grade 1. (B): Tear ferning grade 2. (C): Tear ferning grade 3. (D): Tear ferning grade 4 .

STT values for eyes with abnormal ferning patterns (types 3 and 4 ) were $10.9 \mathrm{~mm} / \mathrm{min}$ (type 3 ) and $12.4 \mathrm{~mm} / \mathrm{min}$ (type 4 ) for the left eye and $11.3 \mathrm{~mm} / \mathrm{min}$ (type 3 ) and $11.9 \mathrm{~mm} / \mathrm{min}$ (type 4) for the right, these not being significantly different between left and right eye but differing from the normal eyes with STT above $10 \mathrm{~mm} / \mathrm{min}$ significantly with $\mathrm{p}$ values of 0.001 and 0.002 respectively. Time to drying of the tear sample was $3.2 \pm 1.2$ minutes for eyes with STT above $15 \mathrm{~mm} / \mathrm{min}$ and $1.8 \pm 1.6$ minutes for eyes with STT below $15 \mathrm{~mm} / \mathrm{min}(\mathrm{p}<0.05)$.

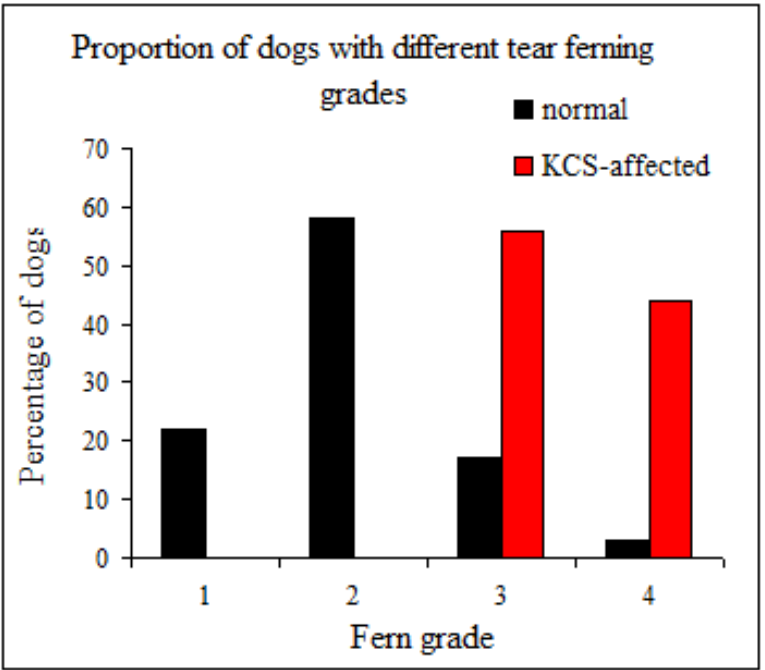

Fig. 3. Proportion of normal and KCS-affected dogs with differing grades of tear film ferning.

Figure 3 shows the percentage of dogs with the four different types of tear ferning in dogs with and without signs of keratoconjunctivitis sicca.

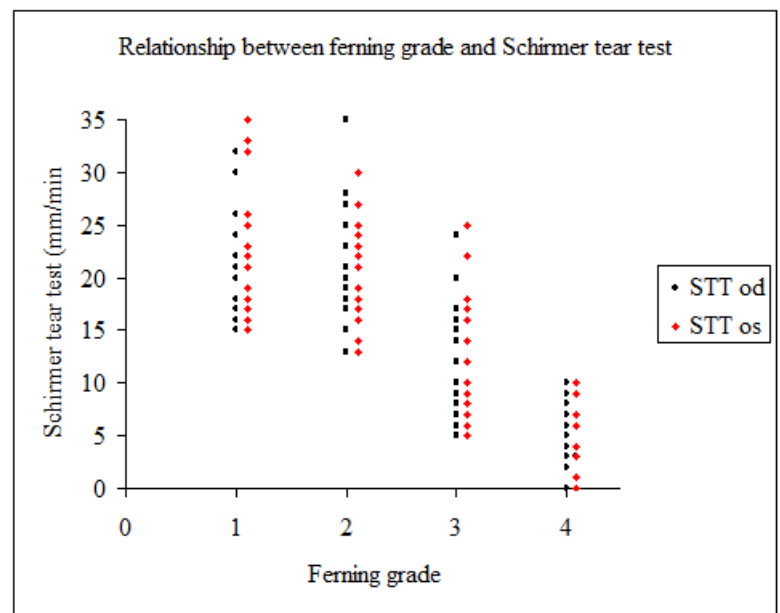

Fig. 4. Graphical representation of the relationship between ferning grade and Schirmer tear test.

Figure 4 shows the correlation between tear ferning pattern and STT value in the dogs evaluated. Mean STT for eyes with ferning types 1,23 and 4 were $22.5 \pm 5.9 \mathrm{~mm} / \mathrm{min}, 20.6 \pm 5.0 \mathrm{~mm} / \mathrm{min}, 11.5 \pm 5.6 \mathrm{~mm} / \mathrm{min}$ and $5.2 \pm 3.2 \mathrm{~mm} / \mathrm{min}$ respectively.

Time to initiation of fern formation was $310 \pm 63$ seconds in dogs with STT greater than $10 \mathrm{~mm} / \mathrm{min}$ and no ophthalmic signs of ocular surface dysfunction and $125 \pm 86$ seconds in dogs with STT lower than $10 \mathrm{~mm} / \mathrm{min}$ and signs of ocular surface pathology such as mucoid discharge, keratitis or ocular surface pigmentation. 


\section{Discussion}

Tear ferning has proved to be a useful tool in the evaluation of the ocular surface in human ophthalmology, in the diagnosis of keratoconjunctivitis sicca and cicatrising keratoconjunctivitis as well as predicting tolerance of the human eye to contact lenses. Here we have shown that tear ferning can be a valuable tool in veterinary ophthalmology too. All tear samples from dogs with keratoconjunctivitis sicca have shown abnormal ferning patterns while a number of animals with apparently normal tear production have shown abnormal ferning patterns. The molecular basis of tear ferning is still somewhat unclear. It may be that mucus is the key to fern production. Mucus lowers tear surface tension facilitating spreading of the tear drop. Tabbara and Okumoto were the first to recognise that tear ferning does not occur in human patients with mucus deficiency (Tabbara and Okumoto, 1982). Norn reported more extensive ferning in human patients with keratitis and bacterial conjunctivitis where mucus production is increased (Norn, 1987), however he also showed that ferning does not relate to the presence of mucus in the tear film on the ocular surface and that tear ferns do not stain with alcian blue and rose bengal, molecules interacting with mucus components (Norn, 1994). Kogbe et al. (1991) showed that a biopolymer is necessary for fern formation but that this need not be mucus.

It is widely accepted that electrolytes are essential for ferning in biological fluids. Ferning occurs in cervical mucus when organic matter is removed but Zondek (1959) found that dialysed cervical mucus, with electrolytes removed, will not form a ferning pattern until electrolytes are replenished. Indeed the same researcher reported demonstrating ferning in solutions of sodium chloride (Zondek, 1959) but that finding has not been repeated by other workers. Increased osmolarity in tear samples from human patients with keratoconjunctivitis sicca may explain the different ferning patterns in those cases and Rolando specifically investigated the effect of hyperosmolarity on tear ferning in samples from human patients, finding that experimental increases in tear osmolarity by adding sodium chloride to the inferior conjunctival sac caused deterioration in grade of tear ferning.

In vitro dilution of tear samples with sodium chloride changed the ferning pattern from grades I and II to grades III and IV but adding glucose to increase the osmolarity did not have the same effect (Rolando, 1988); clearly it is not osmolarity alone which is the important factor but perhaps electrolyte concentration which is more critical to the ferning phenomenon. Protein concentration may be an important factor. Body fluids with a high protein concentration do not exhibit a ferning pattern unless diluted with electrolyte solutions (Zondek and Rozin, 1954). Electrophoretic assessment of tear protein profile and correlation with tear ferning suggested that there is an association with protein concentration (Kogbe and Liolet, 1987). The same research group also showed that tear proteins have an important effect on tear ferning by lowering surface tension of the drop in the same manner that mucus does (Kogbe et al., 1991). In one in vitro experiment stark tear crystal skeletons formed by mixing electrolyte solutions only turn to classic fern patterns when protein is added, but only in a limited range of salt-to-protein ratios. Since electrolyte concentrations in tears from patients with KCS are increased and tear protein concentrations are decreased (Kogbe et al., 1991), this critical ratio for tear fern formation may be the central factor in fern formation. But the very constituents of the electrolyte mix may be critical. The ratio of monovalent sodium and potassium ions to divalent calcium and magnesium ions may also be important, this being suggested by the study conducted by Kogbe et al. (1991). Sequestration of divalent cations with EDTA inhibits fern formation. The truth is that tear ferning is probably influenced by several interacting moieties in the tear film, as suggested by Golding and Brennan (1989), rather than any one in isolation.

A critical paper is that of Pearce and Tomlinson (2000) who used scanning electron microscopy and energy dispersive X-ray analysis to analyse the chemical composition of human tear ferns. Sodium, potassium and chloride were all detected within the fern structure as was sulphur, indicative of the presence of mucin and/or protein, but this latter element was only found at the periphery of the dried tear-film drop. Earlier analysis by X-ray fluoresecence also detected sodium, potassium, chlorine, calcium and sulphur (Golding et al., 1994) but electron microscopy by the same group showed crystalline fern patterns surrounded by globular structures, presumed to be of protein and/or mucin composition. A hypothesis to explain the generation of tear film ferning might thus suggest that as the tear drop dries with water evaporating the electrolyte solution reaches maximal solute concentration and that the proteins are deposited at the drop margin. As evaporation proceeds the solubility limit of electrolytes is reached and ferning proceeds. Given that the protein and mucins have already been deposited at the drop boundary the ferning proceeds unhindered (Pearce and Tomlinson, 2000). The shift in the ratio of electrolytes and macromolecule in eyes with keratoconjunctivitis thus may be the key factor inhibiting fern formation. This hypothesis, however, has been generated with studies of human tear film ferning and it still has to be shown that the equivalent is occurring in the companion animal species.

Collecting tears can be difficult, especially in dogs with a pathologically lower tear production. The animal in 
Figure 1 for instance had a mucoid discharge in the medial canthus associated with an STT value of $9 \mathrm{~mm} / \mathrm{min}$ in that eye. It might be argued that this should be wiped away before collecting a lacrimal sample and this might well be a valuable topic for further study of this technique. In that particular dog the capillary tube was held a millimeter away from the mucus itself to avoid the discharge abnormally affecting the ferning result. As with all tests, familiarity with sampling improves the ease with which one can obtain samples for the tear ferning test. Given the results we have obtained in this study we would recommend that tear ferning be used as an evaluative technique in all cases of canine KCS and would encourage other ophthalmologists to share their experience with this new technique.

\section{Conclusion}

This study has shown that tear ferning occurs with canine tear samples and that the pattern of ferning correlates with tear production as measured by the Schirmer tear test. The correlation is not exact, with some apparently normal tear films yielding abnormal ferning patterns. It may be that tear ferning yields additional information on tear film health not provided by the Schirmer tear test and that tear ferning can be a valuable technique in the evaluation of ocular surface pathology in the dog.

\section{Conflict of interest}

The authors declare that they have no competing interests.

\section{References}

Coassin, M., Lambiase, A., Costa, N., De Gregario, A., Squelleta, R., Sachetti, M., Aloe, L. and Bonini, S. 2005. Efficacy of nerve growth factor treatment in dogs affected by dry eye. Graefes Archiv. Clin. Exp. Ophthalmol. 243, 151-155.

Gelatt, K.N., Peiffer, R.L. Jr., Erickson, J.L. and Gum, G.G. 1975. Evaluation of tear formation in the dog, using a modification of the Schirmer tear test. J. Am. Vet. Med. Assoc. 166, 368-370.

Golding, T.R. and Brennan, N.A. 1989. The basis of tear ferning. Clin. Exp. Optom. 72, 102-112.

Golding, T.R., Baker, A.T., Rechberger, J. and Brennan, N.A. 1994. X-ray and scanning electron microscopic analysis of the structural composition of tear ferns. Cornea 13, 58-66.

Guida, M., Barbato, M., Bruno, P., Lauro, G. and Lampariello, C. 1993. Salivary ferning and the menstrual cycle in women. Clin. Exp. Obstet. Gynecol. 20, 48-54.

Kogbe, O. and Liolet, S. 1987. An interesting use of the study of tear ferning patterns in contactology. Ophthalmologica 194, 150-153.

Kogbe, O., Liotet, S. and Tiffany, J.M. 1991. Factors responsible for tear ferning. Cornea 10, 433-444.
Newcombe, R.G. and Duff, G.R. 1987. Eyes or patients? Traps for the unwary in the statistical analysis of ophthalmological studies. Brit. J. Ophthalmol. 71, 645-646.

Norn, M. 1987. Ferning in conjunctival-cytologic preparations. Crystallisation in stained semiquantitative pipette samples of conjunctival fluid. Acta Ophthalmol. 65, 118-122.

Norn, M. 1988. Quantitative tear ferning. Methodologic and experimental investigations. Acta Ophthalmol. 66, 201-205.

Norn, M. 1994. Quantitative tear ferning. Clinical investigations. Acta Ophthalmol. 72, 369-372.

Papanicolau, G. 1946. A general survey of the vaginal smear and its use in research and diagnosis. Am. J. Obstet. Gynecol. 11, 30-37.

Pearce, E.I. and Tomlinson, A. 2000. Spatial location studies on the chemical composition of human tear ferns. Ophthal. Physiol. Opt. 20, 306-313.

Reece, E.A., Chervenak, F.A., Moya, F.R. and Hobbins, J.C. 1984. Amniotic fluid arborization: effect of blood, meconium, and $\mathrm{pH}$ alterations. Obstet. Gynecol. 64, 248-250.

Rolando, M. 1984. Tear mucus ferning test in normal and keratoconjunctivitis sicca eyes. Chib. Int. J. Ophthalmol. 2, 32-41.

Rolando, M. 1988. Tear mucus crystallization in children with cystic fibrosis. Ophthalmologica 197, 202-206.

Rolando, M., Baldi, F. and Calabria, G. 1986. The effect of hyperosmolarity on tear mucus ferning. Fortsch. Ophthalmol. 83, 644-646.

Silva, L.R., Gouveia, A.F., de Fátima, C.J., Oliveira, L.B., Reis, J.L. Jr., Ferreira, R.F., Pimentel, C.M. and Galera, P.D. 2016. Tear ferning test in horses and its correlation with ocular surface evaluation. Vet. Ophthalmol. 19, 117-123.

Tabbara, K.F. and Okumoto, M. 1982. Ocular ferning test. A qualitative test for mucus deficiency. Ophthalmology 89, 712-714.

Ullery, J.C., Livingstone, N. and Aboushabanah, E.H. 1959. The mucous fern phenomenon in the cervical and nasal smears; a review and current concept of arborization. Obstet. Gynecol. Surv. 14, 1-25.

Van Bijsterveld, O.P. 1990. Diagnosis and differential diagnosis of keratoconjunctivitis sicca associated with tear gland degeneration. Clin. Exp. Rheumatol. 8(Suppl. 5), 3-6.

Williams, D.L. 2008. Immunopathogenesis of keratoconjunctivitis sicca in the dog. Vet. Clin. N. Am. Sm. Anim. Pract. 38, 251-268.

Zondek, B. 1959. Arborization of cervical and nasal mucus and saliva. Obstet. Gynecol. 13, 477-481.

Zondek, B. and Rozin, S. 1954. Cervical mucus arborisation. Its use in the determination of corpus luteum function. Obstet. Gynecol. 3, 463-470. 